\title{
HSV-1-derived amplicon vectors: recent technological improvements and remaining difficulties - A Review
}

\author{
Alberto Luis Epstein \\ Université de Lyon, Université Claude Bernard Lyon 1, Centre de Génétique Moléculaire et Cellulaire, CNRS-UMR5534, 16 rue Raphaël \\ Dubois 2nd floor, F-69622 Villeurbanne, France
}

\begin{abstract}
Amplicons are defective and non-integrative vectors derived from herpes simplex virus type 1. As the vector genome carries no virus genes, amplicons are both non-toxic for the infected cells and non-pathogenic for the inoculated organisms. In addition, the large transgenic capacity of amplicons, which allow delivery of up to $150 \mathrm{Kbp}$ of foreign DNA, makes these vectors one of the most powerful, interesting and versatile gene delivery platforms. We present here recent technological developments that have significantly improved and extended the use of amplicons, both in cultured cells and in living organisms. In addition, this review also discusses the many difficulties still pending to be solved, in order to achieve stable and physiologically regulated transgene expression.
\end{abstract}

Key words: viral vectors - HSV-1-based amplicons - applications - technological improvements

The improvement of methods for efficient delivery and regulated expression of genetic material in mammalian cells has been a major objective of molecular and cellular biology, gene therapy and vaccine development over the last 25 years and it is still an area of intensive research. Viral-derived vectors are the most promising gene transfer tools due to the fact that viruses are naturally occurring molecular devices that have evolved to ensure targeted gene delivery and efficient expression in most cell types.

Herpes simplex virus type 1 (HSV-1)-based vectors have the capacity to deliver up to $150 \mathrm{Kbp}$ of foreign DNA to the nucleus of most proliferating and quiescent mammalian cells, making this family of vectors a very interesting tool for gene transfer and gene therapy. The uniqueness of HSV-1-based vectors stems from several properties of HSV-1: (i) the very large capacity of the virus particle, (ii) the virus DNA will not integrate into host chromosomes, thus reducing the risk of insertional mutagenesis, (iii) the complexity of the virus genome, which contains approximately 40 genes that are not essential for virus replication and can therefore be deleted without disturbing virus production in cultured cells and (iv) the capacity of HSV-1 to infect the nervous system, including the ability to trans-synaptically spread from neuron to neuron in both anterograde and retrograde directions and the capacity to establish latent infections in neurons.

Three different types of vectors can be derived from HSV-1 that attempt to exploit one or more of these properties (Fig. 1). Recombinant attenuated viruses are repli-

Financial support: European Commission (THOVLEN FP6, HEVAR FP6)

Corresponding author: epstein@cgmc.univ-lyon1.fr

Received 15 May 2009

Accepted 15 May 2009 cation-competent HSV-1 vectors carrying mutations that restrict spread and lytic viral replication to cancer cells without causing major toxicity to the healthy tissues. They are used mainly as oncolytic viruses. Defective, replication-incompetent non-pathogenic recombinant HSV-1 vectors lack one or more essential replication genes, but retain many advantageous features of wildtype HSV-1, particularly the ability to express transgenes after having established latent infections in central and peripheral neurons. Amplicon vectors are defective, helper-dependent vectors that carry no viral genes and take advantage of the large carrier capacity of the virus particle to deliver long transgenic sequences. The present review focuses on amplicon vectors, which constitute one of the most powerful and versatile virus-derived gene transfer vectors.

Amplicon vectors (Spaete \& Frenkel 1982) are HSV-1 particles identical to wild-type HSV-1 from the structural, immunological and host-range points of view, but which carry a concatemeric form of a DNA plasmid, named the amplicon plasmid, instead of the viral genome (Fig. 2). HSV-1 particles are composed of approximately 40 different virus-encoded structural proteins. These proteins are delivered into the cell during infection and can trigger cell signalling and cellular responses and, consequently, may have a transient impact on cell homeostasis or gene expression. However, these proteins soon disappear and the cells resume their normal functions, including the ability to divide and to respond to physiological stimuli. An amplicon plasmid (Fig. 2A) is a standard Escherichia coli plasmid carrying one origin of virus replication (OriS) and one packaging signal (pac) from HSV-1, in addition to the transgenic sequences of interest (Vlazny \& Frenkel 1981, Spaete \& Frenkel 1985, Deiss et al. 1986). One major advantage of amplicons as gene transfer tools is the fact that they carry no virus genes and consequently do not induce synthesis of virus proteins. Therefore, these vectors are fully non-toxic for the infected cells and non-pathogenic 
for the transduced organisms. Furthermore, the absence of virus genes in the amplicon genome strongly reduces the risk of reactivation, complementation or recombination with latent or resident HSV-1 genomes.

Amplicons are quite versatile tools due to the fact that during their production, the amplicon genome will replicate, like HSV-1, via a mono-directional, rolling circle-like mechanism, generating long concatemers composed of tandem repeats of the amplicon plasmid (Boehmer \& Lehman 1997) (Fig. 2B). Since infectious HSV-1 particles will always package approximately 150 $\mathrm{Kbp}$ DNA (the size of the virus genome), the number of repeats that a particular amplicon vector will carry and deliver will depend on the size of the original amplicon plasmid (Kwong \& Frenkel 1984). Therefore, an amplicon plasmid of around $5 \mathrm{Kbp}$ will be repeated approximately 30 times in the amplicon vector, while a very large amplicon plasmid, carrying a $150 \mathrm{Kbp}$ genomic locus, will originate amplicon vectors carrying a single repeat of this sequence. Actually, a second major benefit that arises from the lack of virus genes in the amplicon plasmid is that most of the $150 \mathrm{Kbp}$ capacity of the HSV-1 particle can be used to accommodate very large pieces of foreign DNA. This is undoubtedly the most outstanding property of amplicons, as there is no other available viral vector system displaying the capacity to deliver such a large amount of foreign DNA to the nuclear environment of mammalian cells, apart from amplicons derived from other herpesviruses, such as Epstein-Barr virus (EBV) or human cytomegalovirus (HCMV).

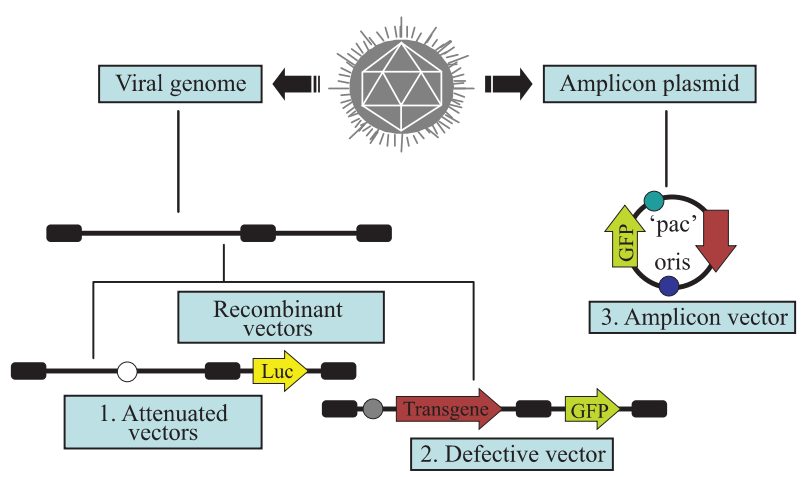

Fig. 1: the three types of HSV-1-based vectors. Recombinant vectors are HSV-1 particles carrying an engineered HSV-1 genome. Recombinant vectors can be attenuated (1), in which case they carry a mutation in a gene affecting virulence (white circle) and generally a reporter gene (in this case luciferase, yellow arrow) to facilitate following the spread of the virus, or defective (2), in which case they carry a deletion in at least one gene encoding an essential function (grey circle). Defective vectors generally carry a transgene of interest (red arrow) and a reporter gene (in this case GFP, green arrow) to identify the infected cells. Amplicon vectors (3) are HSV-1 particles carrying a head-to-tail concatemer of a DNA derived from the amplicon plasmid, instead of the virus genome. These plasmids also generally carry a reporter gene and a transgene of interest. In addition, they carry one HSV-1 origin of DNA replication (oriS) and one packaging signal (pac) to allow amplification and packaging of the plasmid into HSV-1 particles.
In this review we will describe the current state of the art of amplicon vector technology, discuss the present limitations of these vectors and illustrate possible ways currently being investigated to improve the potential of these very interesting gene transfer tools.

\section{Production of amplicon vectors}

Production of amplicon vectors involves the coordinated action of more than 50 viral proteins required to allow replication and packaging of the amplicon plasmid into fully infectious virus particles. The viral genes encoding these proteins could be supplied in principle by a helper virus, by a viral genome or by a packaging cell line. Although it is theoretically possible to construct a packaging cell line able to express the required proteins using a strict inducible system, no such cells are yet available. Hence, the trans-complementing systems currently in use are based either on cloned viral DNA or on helper HSV-1. A major limitation, long associated with the use of amplicons, is the difficulty in producing large, high-titre stocks of vector particles free of helper virus contamination, and the improvement of methods to produce such stocks has been the concern of many studies over the past decade.

The two major methods currently used for producing amplicons, one based on infection with a HSV-1 helper virus and the other based on transfection of HSV-1 genes, are described in Figs 3 and 4, respectively. According to the first method, which has long been the only method available, cells were transfected with the amplicon plas-

A

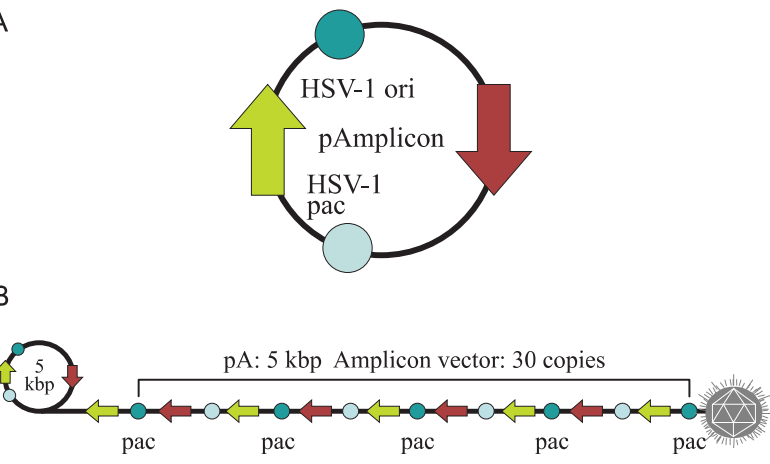

B
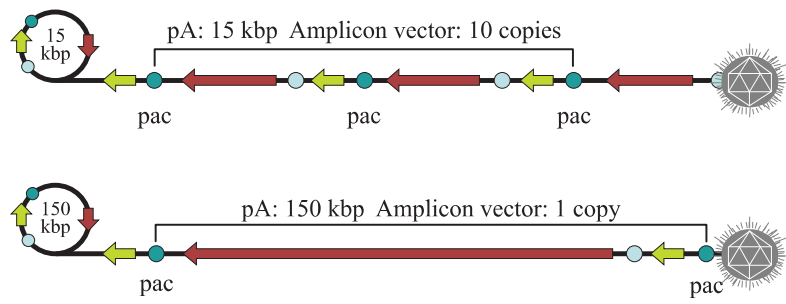

Fig. 2: amplicon plasmid and amplicon vectors. A: an amplicon plasmid is a standard Escherichia coli plasmid carrying one Herpes simplex virus type 1 (HSV-1) origin of DNA replication (oriS), one HSV-1 packaging signal (pac) and the transgenic and reporter sequences of interest (here represented as coloured arrows); B: an amplicon vector is an HSV-1 particle carrying around $150 \mathrm{kbp}$ of a head-to-tail concatemer of DNA derived from the amplicon plasmid. 


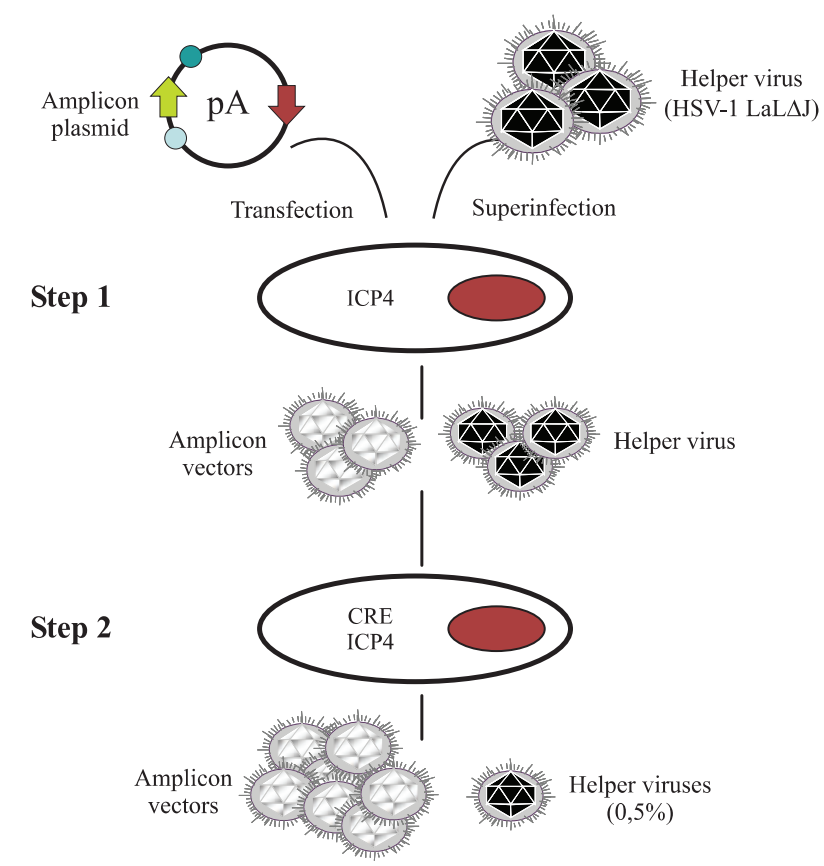

Fig. 3: HSV-1-based method for amplicon vector production. Step 1: after transfection of ICP4-transcomplementing cells with the amplicon plasmid, the viral proteins required for the replication and packaging of this plasmid are supplied by superinfection with HSV-1 LaL $\Delta \mathrm{J}$. This results in the production of helper contaminated amplicon stocks where the ratio of amplicon to helper particles is around 1:1; Step 2: cells expressing both ICP4 and Cre recombinase are infected with the contaminated amplicon stock produced in Step 1. Cre recombinase will induce deletion of the unique packaging sequence of the HSV-1 $\mathrm{LaL} \Delta \mathrm{J}$ virus genome, thus avoiding its packaging. This results in the production of amplicon vector stocks only barely contaminated with replication defective HSV-1 LaL $\Delta \mathrm{J}$ helper particles (ratio of amplicon to helper particles is 100:1-1000:1).

mid then superinfected with a helper HSV-1. The major problem with this method was the production of a mixed population of amplicon vectors and HSV-1 helper particles, which induced strong cytotoxicity upon infection of target cells. Since amplicon vectors and HSV-1 helper particles have identical structural and immunological properties, it is impossible to separate these two types of particles. Therefore, different strategies have been successively developed in order to reduce the toxicity of the helper-contaminated amplicon stocks, either by modifying the helper genome in order to limit its toxicity, or by limiting the production of the contaminant particles.

Historically, the first approach to improve this method involved the use of a thermosensitive HSV-1 as a helper (Geller 1988), but such stocks were unsuitable for gene therapy. Subsequently, defective helper HSV-1, carrying deletions in immediate early genes encoding one essential protein, either ICP4 (Geller et al. 1990) or ICP27 (Lim et al. 1996) were used and produced in complementing cell lines expressing the ICP4 or ICP27 proteins, respectively. A different strategy consisted of the trans-complementation of a function lacking in the helper virus by the amplicon vector itself, thus creating a

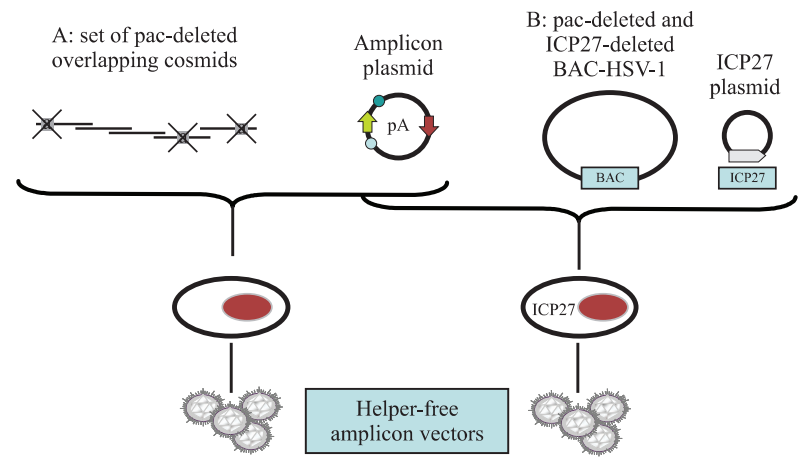

Fig. 4: DNA-based methods for amplicon vector production. A: a set of five overlapping cosmids, deleted for the packaging signals (pac), is cotransfected with the amplicon plasmid in mammalian cells. This allows the generation, by homologous recombination between the overlapping cosmids, of a virus genome able to express all virus proteins but that cannot be packaged into Herpes simplex virus type 1 (HSV-1) particles, as well as a stock of amplicon vectors; B: a bacterial artificial chromosome (BAC)-HSV-1 genome deleted for the packaging signals and for the gene encoding the essential ICP27 protein is cotransfected with both the amplicon plasmid and a plasmid encoding ICP27, in ICP27 expressing cells. As the BAC-HSV-1 genome has been designed to be oversized, this genome cannot be packaged into HSV-1 particles, even in case of recombination with the amplicon plasmid, thus allowing the production of fully helper-free amplicon vectors.

self-complementing binary virus system. This approach was used with defective helper viruses with deletions in genes encoding ICP4 (Pechan et al. 1996) or thymidine kinase (Zhang et al. 1998). However, and despite obtaining relatively high titres, amplicon stocks were still contaminated with HSV-1 helper particles, resulting in significant cytotoxicity. Furthermore, wild-type HSV-1 viruses were often generated by recombination between the amplicon and the helper genomes.

More recently, a third generation of the helper virus system, designed to avoid or limit the packaging of the helper genome was developed. This system is based on the deletion of the pac signals of the helper genome by Cre/loxP-based site-specific recombination, in order to inhibit its encapsidation in the cells that are producing the amplicons. The first such helper system, named HSV-1 LaL helper, carried a unique and ectopic pac signal, flanked by two loxP sites in parallel orientation, located in the gC locus (Logvinoff \& Epstein 2000). This is a Cre-sensitive virus that cannot, in principle, be packaged in Cre-expressing cells due to a deletion of the floxed pac signal. Nevertheless, some helper genomes can escape the action of the Cre recombinase, allowing the production of some helper particles, which are replication-competent and able to spread. To further improve this helper system, the two genes surrounding the pac signal, encoding a virulence factor known as ICP34.5 and the essential protein ICP4, were deleted from the $\mathrm{LaL}$ genome, generating the $\mathrm{LaL} \Delta \mathrm{J}$ helper virus (Zaupa et al. 2003). Although the amplicon stocks prepared with this helper (in a cell line expressing both Cre and ICP4 
proteins) still contain a small amount of contaminating helper particles, this helper is replication-incompetent and cannot spread upon infection of target cells or tissues. Use of the HSV-1 LaL $\Delta \mathrm{J}$ helper virus generally results in the production of large stocks of amplicon vectors only barely contaminated $(0.05-0.5 \%)$ with defective, non-pathogenic helper particles (Fig. 3). Nevertheless, the presence of contaminating helper virus, even at very low levels, could be a limitation for the use of amplicon stocks in some gene therapy applications. This system therefore needs to be further improved.

The second method of amplicon production that allows very low levels or no contamination at all with helper particles is based on cotransfection of amplicon plasmids with HSV-1 genomes after deletion of their packaging signals (Fig. 4). These systems initially derived from a set of five overlapping cosmids, each carrying a large fragment of the virus genome, that allows the reconstitution, by homologous recombination, of wild-type HSV-1 in cells cotransfected with the cosmid set (Cunningham et al. 1993). By deleting the pac sequences from the cosmids, this system can generate a virus genome able to express all the transacting functions but that cannot be packaged into HSV-1 particles. The cotransfection of amplicon plasmids with the modified set of cosmids (Fig 4A) allows the production of amplicon vectors only poorly contaminated with helper particles, which can arise from recombination between the reconstituted helper genome and the amplicon plasmid (Fraefel et al. 1996). The system had been later simplified and significantly improved by cloning the entire HSV-1 genome, lacking however the pac signals, into bacterial artificial chromosomes (BACs) (Stavropoulos \& Strathdee 1998, Saeki et al. 2001, Horsburgh et al. 1999). In the last version of this system, the gene encoding the essential ICP27 protein was further deleted from the BAC-HSV-1 genome, which was then increased in size to further reduce the probability of packaging into newly assembled HSV-1 particles (Saeki et al. 2001, 2003) (Fig. 4B). Th1 ICP27 protein is supplied in trans by both a plasmid and by complementing cell lines. This system allowed the production of entirely helper-free amplicon stocks for the first time. However, the amount of total amplicon vectors produced by this method is limited, as these stocks are produced by transfection procedures and cannot be further amplified.

To conclude, no method combining a total absence of contaminating helper particles with the ability to produce very large amounts of amplicon particles is yet available. Further work aiming to improve production of amplicons therefore has to be pursued in order to overcome these limitations.

\section{Applications of amplicon vectors}

Helper-free amplicon vectors are being used in a large set of experimental anti-cancer strategies and in gene therapy models of neurological disorders. They are also used as tools for fundamental research and in vaccine development. It is not possible to fully describe these applications in the short scope of this review; therefore, the discussion will be limited to the most interesting cases. For a more detailed description of amplicon vector applications, see Cuchet et al. (2007).

Cancer - Amplicons have been used in most anticancer strategies, as shown in Fig. 5 and recently reviewed (Shah \& Breakefield 2006, Cuchet et al. 2007). Since these vectors can efficiently deliver genes to cancer cells but are diluted during successive cell divisions, most studies have used acute approaches, such as antiangiogenic therapy, immunotherapeutic approaches, indirect cell killing using pro-drugs together with vectors expressing pro-drug-modifying proteins, or direct cell killing using vectors expressing a variety of anti-tumour gene products (proteins or shRNA).

Gene therapy - Most studies in this domain have focused on neuroprotection against brain injuries, including the use of amplicons expressing neurotrophic factors, anti-apoptotic proteins, neurotransmitters or neuroreceptors and antioxidant enzymes. A few studies have addressed the use of amplicons for gene therapy of neurodegenerative disorders, such as treatment of experimental models of Parkinson's disease, or inherited genetic diseases affecting the nervous system such as ataxia-telangiectasia and Friedreich's ataxia. These applications are shown in Fig. 6 and reviewed in Tyler et al. (2006) and Cuchet et al. (2007).

Vaccines - Wild-type HSV-1 is a potent immunogen that can elicit both host innate and adaptive immune responses (Whitley 2001), but is also able to evade host immunity due to immunomodulatory gene products such as $v h s$ or the ICP47 protein (York et al. 1994, Samady et al. 2003). The biological characteristics of HSV-1 amplicons that make them an attractive candidate for vaccine delivery applications are safety profile, lack of expression of viral immunoevasive genes, large insert capacity and ability to infect a wide range of cells, including antigen-presenting cells. To date, immunization studies involving amplicons have been essentially directed against viral pathogens, like HIV, and neurological disease fac-

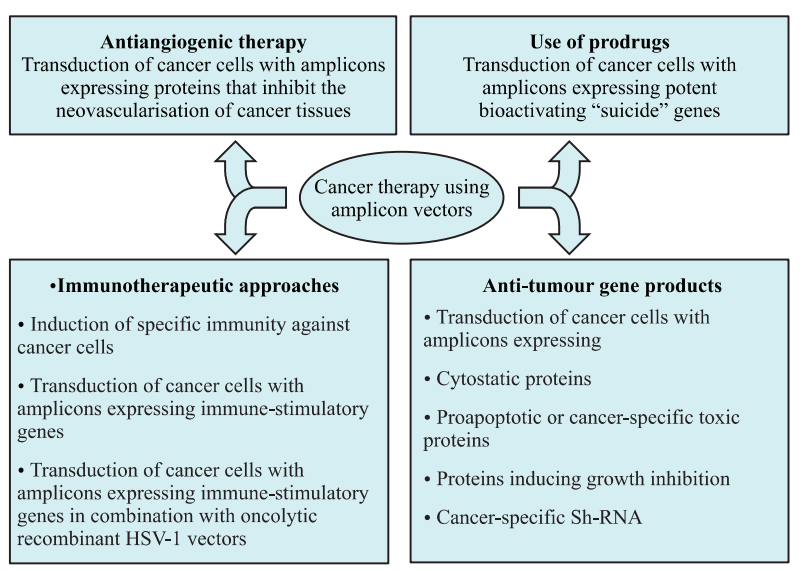

Fig. 5: summary of anti-cancer strategies using amplicon vectors. 


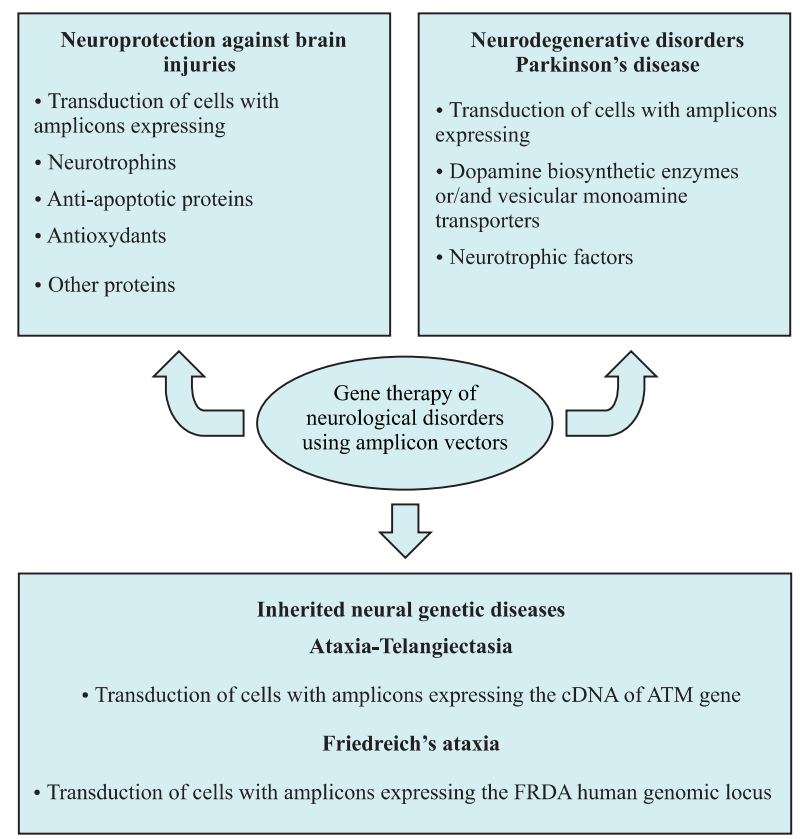

Fig. 6: summary of gene therapy strategies using amplicon vectors.

tors, including Alzheimer's disease and prion disorders. These studies have been recently reviewed (Santos et al. 2006, Tyler et al. 2006, Cuchet et al. 2007).

Due to the high prevalence of HSV infection within the human population, one major concern about their use as vaccines is the possible impact of pre-existing antiviral immunity on vaccine efficiency. This question remains controversial. Some studies, using replication-defective HSV-1, showed strong immune responses even in the presence of anti-HSV immunity (Brockman \& Knipe 2002, Hocknell et al. 2002). In contrast, another study with replication-defective HSV-1 showed a substantial reduction in immune responses in animals previously vaccinated against HSV-1 (Lauterbach et al. 2005).

Fundamental studies - In addition to the therapeutic applications of amplicons, many studies have used these vectors for fundamental studies. Thus, amplicon vectors have been delivered into distinct brain regions to investigate many aspects of the functioning of the central nervous system, including the molecular and cellular basis of functions such as behaviour and cognition, neuroplasticity and neurogenesis, as recently reviewed (Jerusalinsky \& Epstein 2006, Cuchet et al. 2007). Many of these studies took advantage of previous works that demonstrated the potential of amplicons carrying reporter genes as gene delivery tools to the nervous system (Ho et al. 1993, During et al. 1994, Brooks et al. 2000).

Amplicons have also been used to induce the synthesis of one or more proteins from other virus species, in order to study their function, to generate hybrid vectors or to elicit immune responses. In the more complex settings, amplicons expressing the full set of structural proteins from other viruses allowed the assembly of empty virus-like particles, including retrovirus (Savard et al. 1997, Parrish et al. 1999, Sena-Esteves et al. 1999) and hepatitis C virus (Hong et al. 1996, Tsitoura et al. 2002, 2006, Kalamvoki \& Mavromara 2004) particles, which can be eventually useful either as vaccines or as vectors in specific protocols of gene therapy.

\section{Unresolved questions}

The above paragraphs illustrate the very strong potential of amplicons as gene delivery vectors for fundamental and applied studies. However, our knowledge on many aspects of amplicon biology remains fragmentary and many technological problems are still unresolved. This last chapter addresses what is perhaps the most important still unresolved problem: the transient nature of expression of the transgenic cassettes delivered by amplicons, which arises both from instability of the transgenic cassette and from silencing of its expression in the target cells. A different but perhaps linked problem, related to the innate and adaptive immune responses elicited by entry of the amplicon or expression of the transgenic proteins, is only now beginning to be investigated.

\section{Expression of the transgenic cassettes}

Expression of transgenes delivered by amplicon vectors must be analyzed considering the following aspects: tissue specificity, physiological regulation, intensity and length of expression. In this and the following chapters, we will focus only on studies designed to improve gene delivery and expression using amplicon vectors free of helper contamination. In fact, some older data should have to be re-analyzed taking into account the fact that they were obtained with helpercontaminated vector stocks and it is clear today that the presence of helper particles strongly affects most parameters of transgenic expression.

Transgenic expression following infection with helper-free amplicon stocks is transient in most experimental settings and the ability to express a given transgene is cell-type specific. Indeed, after infection of dividing cells, the loss of the standard amplicon genomes is fast since there is no vector amplification in the infected cells. However, also in cells that do not divide the standard amplicon genome is only expressed during a relatively short time and this is not related to cell death. Therefore, the amplicon genome probably becomes silenced.

Amplicon infection induces a cascade of innate responses - The strength and duration of helper-free amplicon-mediated transgene (GFP) expression can range from strong expression in nerve or muscle cells, which can last for several days, to very weak or no expression in low-passage human fibroblasts, likely implying the involvement of cellular factors. Since UV-treated HSV-1 and HSV-1 mutants deficient in the expression of some immediate-early proteins, can trigger a strong antiviral response (Mossman et al. 2001, Mossman \& Smiley 2002), knockout mice showed 10 -fold higher transgene (luciferase) expression than in wild-type mice, and luciferase expression remained detectable during at least 80 days, while in wild-type mice luciferase expression became undetectable after two weeks post-infection 
(Suzuki et al 2007). Additional studies using fibroblasts derived from wild-type and STAT1-knockout mice revealed the significance of STAT1 signalling in transcriptional silencing of the amplicon-encoded transgene in vitro, indicating that type I interferons (IFN) induced by systemic delivery of amplicons may initiate a cascade of immune responses that suppress transgene expression at the transcriptional level (Suzuki et al. 2007). In a further study, antiviral responses were investigated following stereotactic amplicon administration into the mouse striatum. In the brain, induction of type I IFN was rather modest and transgene expression lasted over a year, despite inflammation and infiltration of immune cells around the injection sites. These findings indicate that the spectrum of host responses can differ significantly depending on target organs and administration routes (Suzuki et al. 2008).

Amplicon infection of cultured human foetal foreskin fibroblasts (HFFF-2) results in the induction of an interferon regulatory factor 3 (IRF3)-dependent antiviral response, characterized by the upregulation of IRF7 and Toll-like receptor 3 (TLR3), the upregulation of some interferon stimulated genes (ISG), such as ISG54 and ISG56, and the secretion of low levels of $\beta$-IFN. These responses lead to the establishment of an antiviral state in the amplicon-infected cells, which become refractory to subsequent infection with vesicular stomatitis virus (Tsitoura \& Epstein, unpublished observations). However, although GFP expression is 5-20-fold lower in HFFF-2 cells than in human glioma cell lines or in mouse primary cardiomyocytes, this seems not to be caused by the triggering of IRF3-dependent antiviral responses since siRNA-mediated down-regulation of IRF3 before infection, while significantly reducing levels of ISG expression, does not result in the enhancement of transgene expression from the amplicon genome (Tsitoura \& Epstein, unpublished observations).

Extinction of transgene expression - There are no complete answers yet to explain why transgenic expression from an amplicon genome becomes extinct in the absence of helper proteins. The failure to explain the silencing of transgene expression by the induction of IRF3signalled innate antiviral responses suggest, however, that other types of cellular silencing factors are possibly acting on the vector genome. Recently, a very interesting investigation indicated that bacterial sequences present in the amplicon genome can cause rapid transgene transcriptional silencing by forming inactive chromatin (Suzuki et al. 2006). Indeed, each amplicon vector genome contains several copies of the original plasmid sequence, depending on the size of the amplicon plasmid. Infection with amplicons devoid of bacterial sequences (minicircle amplicons) induced approximately 20 -fold higher transgene expression and quantitative analysis of levels of transgenic mRNA revealed that the increase in transgene expression was at the transcriptional level. In addition, nude mice injected with minicircle amplicons exhibited 10 -fold higher luciferase expression than mice injected with conventional amplicons. Furthermore, luciferase expression from conventional amplicons was undetectable 21 days after injection, whereas with the minicircle amplicons expression was detectable up to at least 28 days post-infection (Suzuki et al. 2006). These observations suggest that inherent cellular proteins, perhaps recognizing naked foreign DNA entering the nucleus, are probably implicated in the silencing of the amplicon genome.

ICP0 is an HSV-1 immediate early regulator protein that plays a major role in virus infection by activating all classes of virus gene expression. Everett and co-workers, and other groups, have shown that ICP0-null HSV-1 mutants can infect foetal human fibroblasts and deliver the virus genome to the cell nucleus, but expression from the virus genome is immediately silenced when the cells are infected at low multiplicity, at least in part due to the silencing action of the promyelocytic leukemia (PML) protein and other nuclear domain 10 (ND10)-related proteins (Everett et al. 2004, 2006). The immediate-early ICP0 protein, which contains an E3-ubiquitin ligase activity (Boutell et al. 2005), allows overcoming this repression by inducing the proteasome-dependent proteolytic degradation of several ND10-related proteins (Chelbi-Alix \& De The 1999, Gu \& Roizman 2003). Since amplicons carry neither ICP0 nor any other viral genes, it is possible that the very low level of transgenic expression observed in human fibroblasts could be caused by the action of these inherent cellular silencing responses. In support of such a hypothesis, amplicons expressing GFP under the control of the IE4/5 HSV-1 promoter and wildtype ICP0 driven by the HCMV promoter expressed higher levels of GFP in human primary fibroblasts, cultured rat cardiomyocytes and rat neonatal cultured brain cells, than amplicons expressing no ICP0 or a mutated inactive form of ICP0, suggesting that ICP0 could play a significant role in suppressing a silencer activity in the amplicon-infected cells. Since, as already quoted, one important role of ICP0 is to induce degradation of several ND10 constitutive proteins that are thought to contribute to silencing of gene expression, including PML and Sp100, these results lend further support to the hypothesis that an inherent antiviral mechanism is partially responsible for the silencing of GFP expression in human fibroblasts. Furthermore, siRNA downregulation of Sp100 and Daxx, two regulatory proteins that localize to PML bodies, resulted in at least 5-fold enhancement in the number of GFP-expressing normal human fibroblasts infected with standard amplicons (Tsitoura \& Epstein, unpublished observations). Lastly, the observation that the reduction of particle-associated ICP0 levels, which results from packaging the amplicon genome in the presence of the transcriptional regulator hexamethylene bisacetylamide, also results in reduced transgene expression (Burris et al. 2008), provides further evidence for a role of ICP0 in suppressing silencing mechanisms that repress GFP expression.

Effect of the promoter on transgenic expression - Most studies with amplicons used viral promoters (generally the HSV-1 IE4/5 promoter or the HCMV IE enhancer/ promoter) to drive transgenic expression. This type of promoter allowed strong expression in many cell types 
but, as already described, expression becomes silenced after a relatively short time period. Several alternatives to viral promoters have been considered, such as the use of inducible promoters, tissue-specific promoters, or a complete genomic locus with endogenous promoters and other regulatory sequences.

Several inducible systems have been developed and used in amplicon vectors, including the glucocorticoidinducible system (Lu \& Federoff 1995), the tetracyclineresponsive promoter system (Ho et al. 1996, Fotaki et al. 1997) and the rapamycin-based "dimerizer" system (Wang et al. 2003). All of these studies showed the feasibility of inserting these systems in amplicons vectors, but all of them showed considerable basal expression. This basal activation is perhaps due to the TAATGARAT DNA motifs present in OriS, which could be activated by VP16, a transactivating HSV-1 protein that is incorporated in the tegument of the amplicon particles during vector production and delivered to the cells upon infection (Lu \& Federoff 1995). The action of such a very strong transcription factor on the amplicon genome could activate the inducible promoters in a non-specific manner.

Regarding the use of tissue-specific promoters, the rat preproenkephalin promoter-driven lac $Z$ expression showed a restricted pattern of transgene expression that persisted in the rat brain for two months after infection (Kaplitt et al. 1994). The tyrosine hydrolase promoter was also used in similar studies and showed cell-specific expression that lasted six weeks (Song et al. 1997). Using these two endogenous promoters, a more restricted and longer transgenic expression was observed as compared with viral promoters.

Physiological regulation of transgenic expression is critical for efficient gene therapy. Accordingly, studies were performed using very long (Gomez-Sebastian et al. 2007, Jeong et al. 2007, Rasmussen et al. 2007) or entire human genomic loci containing the native promoter and very long flanking upstream and downstream sequences (Wade-Martins et al. 2003, Hibbitt \& Wade-Martins 2006), exploiting the large transgenic capacity of amplicons. These amplicons showed good physiological expression of the human low-density lipoprotein receptor gene (LDLR) (Wade-Martins et al. 2003) and of the FRDA gene (Gomez-Sebastian et al. 2007) in proliferating cells.

\section{Maintenance of the transcription cassette in divid- ing cells}

In addition to the transcriptional silencing of the transgenic sequences, long-term expression from standard amplicons cannot be attained in dividing cells due to the dilution of the replication-incompetent vector genome. In order to achieve stability of the transgenic cassettes in dividing cells, two very distinct strategies are currently being developed. One of them attempts to confer longterm expression via the integration of the transgenic cassette into host chromosomes. The other strategy aims to confer long-term expression via the conversion of the amplicon genome into a replication-competent extrachromosomal element.

\section{Toward the integration of the transgenic sequences}

HSV-1/adeno-associated virus system (AAV) hybrid amplicons - In order to increase the length and efficiency of transgene expression, chimeric amplicon vectors carrying elements of the $\mathrm{AAV}$ are being investigated for their ability to induce integration of the transgenic cassette into specific sites of host chromosomes.

The HSV-1/AAV hybrid amplicon vectors carry two genetic elements from AAV, the inverted terminal repeat sequences (ITRs) and the rep gene. The Rep protein is expected to promote the integration of the ITR-flanked transgenic sequences carried by the amplicon genome at the AAVS1 site in chromosome 19 in the ampliconinfected human target cells. In initial studies aimed at comparing standard amplicons with HSV-1/AAV hybrid amplicons, Johnston et al. (1997) modified a standard amplicon genome, carrying the reporter lac $Z$ gene, by flanking the transgenic cassette with AAV ITR sequences, either alone or with a transcription unit expressing Rep. After infection of glioma cells with the standard amplicon vector, lac $Z$ expression was lost after 10 days while the hybrid amplicons allowed expression for at least two weeks in the presence of Rep protein (Johnston et al. 1997). Fraefel et al. (1997) also developed an HSV-1/ AAV hybrid amplicon vector expressing a reporter gene under the control of a liver-specific promoter that was used in vivo to infect mouse liver. Analyses of tissue sections also revealed extended transgenic expression when delivered by the hybrid amplicon. Other studies have shown that including AAV ITRs and the rep gene in the amplicon genome enhanced short-term levels of transgenic expression in different types of cells, such as glioblastoma cells (Lam et al. 2002, Wang et al. 2002), primary myoblasts (Wang et al. 2002), human epithelial kidney cells (Heister et al. 2002) and immortalized mouse embryonic fibroblasts (Bakowska et al. 2003); however, it has to be pointed out that in some cell lines, Rep may have toxic effects.

A major problem found with the HSV-1/AAV hybrid amplicons is the very low efficiency of their production. Indeed, as Rep can interfere with HSV-1 replication (Heilbronn et al. 1990) during the production of HSV1/AAV hybrid amplicon vectors, a low packaging efficiency was observed, resulting in very low titres of these amplicons (Heister et al. 2002, Wang et al. 2002). Two strategies were thus developed to improve the production of HSV-1/AAV hybrid amplicons. The first strategy was to replace the HSV-1 origin of replication by 55 sequences of AAV which can act as an origin of replication (Glauser et al. 2005). This new hybrid amplicon genome could be packaged into HSV-1 virions in the presence of HSV-1 helper functions and Rep, whose presence becomes mandatory in this system. The second approach was to design a hybrid vector that allows Rep expression only in the presence of the Cre recombinase to eliminate intervening floxed sequences that block Rep expression. In this system, Rep will not be expressed in the packaging cells, which do not express Cre, but will be expressed in the transduced cells that have to express Cre to delete the intervening sequences. In this case, Rep will be ex- 
pressed, but only until the occurrence of Rep-mediated integration of the transgenic cassette, which results in novel suppression of Rep expression (Liu et al. 2006). Several results using the HSV-1/AAV hybrid amplicons have clearly demonstrated that it is indeed possible to target integration into the AAVS1 site, resulting in sustained expression of the transgenic cassette. However multiple integrations at other sites can also take place, perhaps causing genotoxic events, and this very interesting system therefore needs therefore to be further improved (Heister et al. 2002, Bakowska et al. 2003, Liu et al. 2006). For an exhaustive review on HSV-1/AAV hybrid amplicons, please refer to Glauser et al. (2006).

Transposons - A second strategy to induce integration into host chromosomes of transgenic sequences delivered by amplicon vectors is the use of transposons. It has been shown that it is possible to induce integration of a transgenic cassette flanked with repeated sequences of the Tcl family of transposons in the presence of a transposase or an integrase (Ivics et al. 1997). Accordingly, a modified amplicon vector that uses the Tcl-like Sleeping Beauty transposon system to create an integrating amplicon vector platform has recently been constructed and tested by Bowers et al. (2006). This amplicon allowed the integration of the reporting transgene and extension of its expression in neuronal precursors. However, in contrast to the HSV-1/AAV hybrid system, integration of the transgenic cassette is not targeted and can take place at multiple random places.

\section{Toward the maintenance of the amplicon genome as a replicative episome}

Three methods are being used in order to transform the amplicon genome into a replication-competent extrachromosomal element. The first one uses the properties of the EBV replicon, the second uses the properties of cellular scaffold/matrix attachment region (S/MAR) sequences and the third uses the technology of human artificial chromosomes (HACs).

HSV-1/EBV-hybrid amplicons - Hybrid amplicon vectors containing the EBV latent replication origin sequence oriP and expressing a modified EBNA-1 protein allowed nuclear replication and segregation of the amplicon genome in dividing human cells. This system allows for efficient packaging of high-titre vectors that successfully expressed a transgenic cassette in several cancerderived cell lines and in human liver tumour implants in nude mice (Wang \& Vos 1996, Wang et al. 1997). HSV-1/EBV-hybrid amplicon vectors were also used to confer long-term physiological expression of the human hypoxanthine phosphoribosyltransferase (HPRT) in transduced cells. HPRT is an enzyme that catalyzes an early step in the purine salvage pathway. The complete HPRT locus (115 Kbp) was cloned into an HSV-1-EBVhybrid amplicon vector, which was used to infect human HPRT-deficient fibroblast cells and a primary mouse hepatocyte culture derived from $\mathrm{Hprt}^{-/}$mice. In both cases, the authors noted an efficient infectious delivery of the functional genomic DNA locus (Wade-Martins et al. 2001). The authors also demonstrated the persistence of the amplicon episome for at least two months in selected clones of the infected human HPRT-deficient fibroblasts. These studies confirm the interest of using the EBV replicons to maintain amplicon genomes carrying very large DNA fragments in dividing cells. An HSV-1/ EBV-hybrid amplicon vector encoding a secreted reporter protein was compared to its relative standard amplicon vector. Infection of primary murine hepatocyte cultures with these two vectors revealed that the length of gene expression increased from two weeks with standard amplicons to six weeks using the hybrid amplicon. Injection of the hybrid amplicon vectors into the liver of SCID mice also increased the level of transgene expression and its duration for several weeks (Muller et al. 2005). These results suggest that the EBV replicon can also stimulate transgenic expression in quiescent cells.

At least two facts, however, considerably limit the applications of HSV-EBV-hybrid amplicons. First, the EBV replicon works efficiently mainly in human cells and, in most cases, only using selection procedures or strategies that confer any selective advantage to the cells carrying these vectors. Secondly, there is increasing concern with the use of these vectors in gene therapy protocols due to the possible oncogenic potential of the EBNA1 protein (Tsimbouri et al. 2002).

$S / M A R$ - A recent paper described the development of a high capacity episomal vector system exploiting human S/MAR sequences to provide vector maintenance and regulated gene expression through the delivery of a genomic DNA locus using amplicons (Lufino et al. 2007). This system was used to deliver and maintain a $135 \mathrm{Kbp}$ genomic DNA insert carrying the LDLR genomic DNA locus at high efficiency in CHO clonal cells not expressing this receptor (ldlr ${ }^{-/}$a7 cells). Long-term studies on $\mathrm{CHO}$ ldlr $^{-/}$a7 clonal cell lines carrying the S/MAR-LDLR amplicons demonstrated episomal retention of 1-4 vector copies per cell, as estimated by Southern blots, and episomal stability of the vectors for more than 100 cell generations without selection. Expression studies of these cells showed restored LDLR function to physiological levels. Thus, this vector seems to simultaneously overcome the major problems of vector loss and unregulated transgene expression.

$H A C s$ - A third, recently developed alternative to confer stability to episomal amplicons is to convert the amplicon genomes into HACs. HACs are DNA molecules that contain human alphoid DNA sequences, which allow segregation and retention during human cell division (Larin \& Mejia 2002). Traditional methods to deliver HACs involved laborious procedures with low effectiveness and that usually resulted in shearing and degradation of HACs. By introducing alphoid sequences of different lengths into the amplicon plasmid, Moralli et al. (2006) developed a series of HAC amplicon vectors. These tools were used to transduce different human cell lines, including glioma, lung fibroblast and kidney 293 human cells, with a much higher efficacy than transfection-based methods. In fibrosarcoma cells, a HAC amplicon expressing HPRT successfully complemented the HPRT deficiency. It was also shown that the length of expression of 
the HAC was cell-type dependant. Whereas HACs were rapidly lost in kidney 293 and in primary cells (MRC5), they were stable for more than three months in selected fibrosarcoma and glioma cells. Although many improvements to this system are certainly required, the study of Moralli et al. (2006) describes a significant advance in amplicon technology. For a recent review on advances in episomal maintenance and expression of gene delivery vectors, see (Lufino et al. 2008).

\section{Concluding remarks}

Due to their very large transgenic capacity, amplicons are one of the most interesting, versatile, powerful and promising gene transfer platforms. These vectors are able to deliver many copies of a small transgenic cassette or a group of genes encoding the full set of proteins required to assemble complex structures, to express several antigens from a given pathogen or to deliver one copy of a $150 \mathrm{Kbp}$ genomic locus, including all exons, introns and large upstream and downstream regulatory sequences. Amplicons are the only viral vectors available that can deliver human or mice artificial chromosomes to the nuclear environment of mammalian cells. They have been successfully used to deliver transgenic cassettes in different programs of experimental gene therapy, in development of HSV-1-based vector vaccines, and in a large number of fundamental studies.

Many recent technological developments have improved and are extending the use of amplicons. We would like to stress in particular the development of two different, and in some sense complementary, systems to produce vectors devoid of contaminating helper particles or carrying only a very low amount of completely defective and non-pathogenic helper particles. The demonstration that amplicons can be used to deliver very large DNA fragments, which appeared an unattainable dream 10 years ago, is now a reality and many groups today are exploiting this unique property of amplicons in many different experimental settings. The introduction of the MARs or HAC sequences into the amplicon vector and the development of vectors that induce targeted integration into the host chromosomes have recently shown that it is possible to avoid dilution of the transgenic cassette delivered by the amplicons to proliferating cells.

However, we should also stress the many limitations of this system that should be resolved before these vectors can be safely and efficiently applied to human beings in gene therapy or vaccine protocols. The production and purification procedures of amplicon vectors need to be, and probably can be, be further improved. We still do not completely understand the factors that affect control of expression and result in the silencing of the transgenic cassette delivered by amplicons, though the demonstration that the use of long native DNA regulatory sequences can confer long-term physiological control of expression opens a door for the possible resolution of this problem. The systems designed to avoid dilution of the transgenic cassette, with or without integration into host chromosomes, are still imperfect and can certainly be optimized. Several aspects of the biology of amplicons, related in particular to the cellular and host defences against infection or expression of transgenic proteins, are only now beginning to be explored. Research and development on other domains of the amplicon biology or technology is just beginning, including the possible engineering of the tropism of amplicons or the development of other types of hybrid or combined vector systems that could eventually achieve transport and delivery of the transgenic cassettes to regions of the brain that are difficult to access without surgical intervention.

Amplicon research is very dynamic and the very large transgenic capacity of these vectors offers unique possibilities for the resolution of many problems that cannot be done with smaller vector systems. Probably the strongest future challenge that will boost amplicon research and development will be the successful application of these vectors to human beings. At the light of the incredible progress achieved in the last 10 years, we have few doubts in that such an eventuality will arrive rather soon.

\section{ACKNOWLEDGEMENTS}

To the French societies Association pour la Recherche contre le Cancer (ARC), Association Française contre les Myopathies (AFM) and the Saône et Loire Committee of Ligue Nationale contre le Cancer (LNCC), for constant support to our laboratory.

\section{REFERENCES}

Bakowska JC, Di Maria MV, Camp SM, Wang Y, Allen PD, Breakefield XO 2003. Targeted transgene integration into transgenic mouse fibroblasts carrying the full-length human AAVS1 locus mediated by HSV/AAV rep $(+)$ hybrid amplicon vector. Gene Ther 10: 1691-1702.

Boehmer PE, Lehman IR 1997. Herpes simplex virus DNA replication. Annu Rev Biochem 66: 347-384.

Boutell C, Canning M, Orr A, Everett RD 2005. Reciprocal activities between herpes simplex virus type 1 regulatory protein ICP0, a ubiquitin E3 ligase, and ubiquitin-specific protease USP7. J Virol 79: $12342-12354$.

Bowers WJ, Mastrangelo MA, Howard DF, Southerland HA, Maguire-Zeiss KA, Federoff HJ 2006. Neuronal precursor-restricted transduction via in utero CNS gene delivery of a novel bipartite HSV amplicon/transposase hybrid vector. Mol Ther 13: 580-588.

Brockman MA, Knipe DM 2002. Herpes simplex virus vectors elicit durable immune responses in the presence of preexisting host immunity. J Virol 76: 3678-3687.

Brooks AI, Cory-Slechta DA, Bowers WJ, Murg SL, Federoff HJ 2000. Enhanced learning in mice parallels vector-mediated nerve growth factor expression in hippocampus. Hum Gene Ther 11: 2341-2352.

Burris CA, de Silva S, Narrow WC, Casey AE, Lotta LT Jr, Federoff HJ, Bowers WJ 2008. Hexamethylene bisacetamide leads to reduced helper virus-free HSV-1 amplicon expression titers via suppression of ICP0. J Gene Med 10: 152-164.

Chelbi-Alix MK, De The H 1999. Herpes virus induced proteasomedependent degradation of the nuclear bodies-associated PML and Sp100 proteins. Oncogene 18: 935-941.

Cuchet D, Potel C, Thomas J, Epstein AL 2007. HSV-1 amplicon vectors: a promising and versatile tool for gene delivery. Expert Opin Biol Ther 7: 975-995.

Cunningham C, Davison AJ 1993. A cosmid-based system for constructing mutants of herpes simplex virus type 1. Virology 197: 116-124. 
Deiss LP, Chou J, Frenkel N 1986. Functional domains within the a sequence involved in the cleavage-packaging of herpes simplex virus DNA. J Virol 59: 605-618.

During MJ, Naegele JR, O’Malley KL, Geller AI 1994. Long-term behavioral recovery in parkinsonian rats by an HSV vector expressing tyrosine hydroxylase. Science 266: 1399-1403.

Everett RD, Boutell C, Orr A 2004. Phenotype of a herpes simplex virus type 1 mutant that fails to express immediate-early regulatory protein ICP0. J Virol 78: 1763-1774.

Everett RD, Rechter S, Papior P, Tavalai N, Stamminger T, Orr A 2006. PML contributes to a cellular mechanism of repression of herpes simplex virus type 1 infection that is inactivated by ICP0. J Virol 80: 7995-8005.

Fotaki ME, Pink JR, Mous J 1997. Tetracycline-responsive gene expression in mouse brain after amplicon-mediated gene transfer. Gene Ther 4: 901-908.

Fraefel C, Jacoby DR, Lage C, Hilderbrand H, Chou JY, Alt FW, Breakefield XO, Majzoub JA 1997. Gene transfer into hepatocytes mediated by helper virus-free HSV/AAV hybrid vectors. Mol Med 3: 813-825.

Fraefel C, Song S, Lim F, Lang P, Yu L, Wang Y, Wild P, Geller AI 1996. Helper virus-free transfer of herpes simplex virus type 1 plasmid vectors into neural cells. J Virol 70: 7190-7197.

Geller AI 1988. A new method to propagate defective HSV-1 vectors. Nucleic Acids Res 16: 5690.

Geller AI, Keyomarsi K, Bryan J, Pardee AB 1990. An efficient deletion mutant packaging system for defective herpes simplex virus vectors: potential applications to human gene therapy and neuronal physiology. Proc Natl Acad Sci USA 87: 8950-8954.

Glauser DL, Ackermann M, Saydam O, FraefeL C 2006. Chimeric herpes simplex virus/adeno-associated virus amplicon vectors. Curr Gene Ther 6: 315-324.

Glauser DL, Saydam O, Balsiger NA, Heid I, Linden RM, Ackermann M, Fraefel C 2005. Four-dimensional visualization of the simultaneous activity of alternative adeno-associated virus replication origins. J Virol 79: 12218-12230.

Gomez-Sebastian S, Gimenez-Cassina A, Diaz-Nido J, Lim F, WadeMartins R 2007. Infectious delivery and expression of a $135 \mathrm{~kb}$ human FRDA genomic DNA locus complements Friedreich's ataxia deficiency in human cells. Mol Ther 15: 248-254.

Gu H, Roizman B 2003. The degradation of promyelocytic leukemia and $\mathrm{Sp} 100$ proteins by herpes simplex virus 1 is mediated by the ubiquitin-conjugating enzyme UbcH5a. Proc Natl Acad Sci USA 100: 8963-8968.

Heilbronn R, Burkle A, Stephan S, Zur Hausen H 1990. The adenoassociated virus rep gene suppresses herpes simplex virus-induced DNA amplification. J Virol 64: 3012-3018.

Heister T, Heid I, Ackermann M, Fraefel C 2002. Herpes simplex virus type 1/adeno-associated virus hybrid vectors mediate sitespecific integration at the adeno-associated virus preintegration site, AAVS1, on human chromosome 19. J Virol 76: 7163-7173.

Hibbitt OC, Wade-Martins R 2006. Delivery of large genomic DNA inserts $>100 \mathrm{~kb}$ using HSV-1 amplicons. Curr Gene Ther 6: 325-336.

Ho DY, Mc Laughlin JR, Sapolsky RM 1996. Inducible gene expression from defective herpes simplex virus vectors using the tetracycline-responsive promoter system. Brain Res Mol Brain Res 41: 200-209.

Ho DY, Mocarski ES, Sapolsky RM 1993. Altering central nervous system physiology with a defective herpes simplex virus vector expressing the glucose transporter gene. Proc Natl Acad Sci USA 90: 3655-3659.

Hocknell PK, Wiley RD, Wang X, Evans TG, Bowers WJ, Hanke T, Federoff HJ, Dewhurst S 2002. Expression of human immunodeficiency virus type 1 gp120 from herpes simplex virus type 1-derived amplicons results in potent, specific and durable cellular and humoral immune responses. J Virol 76: 5565-5580.

Hong Z, Ferrari E, Wright-Minogue J, Chase R, Risano C, Seelig G, Lee CG, Kwong AD 1996. Enzymatic characterization of hepatitis $\mathrm{C}$ virus NS3/4A complexes expressed in mammalian cells by using the herpes simplex virus amplicon system. J Virol 70: 4261-4268.

Horsburgh BC, Hubinette MM, Qiang D, MacDonald ML, Tufaro F 1999. Allele replacement: an application that permits rapid manipulation of herpes simplex virus type 1 genomes. Gene Ther 6: 922-930.

Ivics Z, Hackett PB, Plasterk RH, Izsvak Z 1997. Molecular reconstruction of Sleeping Beauty, a Tc1-like transposon from fish, and its transposition in human cells. Cell 91: 501-510.

Jeong KH, Bakowska JC, Song IO, Fu N, Brakefield XO, Kaiser UB 2007. Improvement in reproductive parameters in hypogonadal female mice by regulated gene replacement therapy in the central nervous system. Gene Ther 14: 1092-1101.

Jerusalinsky D, Epstein AL 2006. Amplicon vectors as outstanding tools to study and modify cognitive functions. Curr Gene Ther 6: $351-360$.

Johnston KM, Jacoby D, Pechan PA, Fraefel C, Borghesani P, Schuback D, Dunn RJ, Smith FI, Breakefield XO 1997. HSV/AAV hybrid amplicon vectors extend transgene expression in human glioma cells. Hum Gene Ther 8: 359-370.

Kalamvoki M, Mavromara P 2004. Calcium-dependent calpain proteases are implicated in processing of the hepatitis $\mathrm{C}$ virus NS5A protein. J Virol 78: 11865-11878.

Kaplitt MG, Kwong AD, Kleopoulos SP, Mobbs CV, Rabkin SD, Pfaff DW 1994. Preproenkephalin promoter yields region-specific and long-term expression in adult brain after direct in vivo gene transfer via a defective herpes simplex viral vector. Proc Natl Acad Sci USA 91: 8979-8983.

Kwong AD, Frenkel N 1984. Herpes simplex virus amplicon: effect of size on replication of constructed defective genomes containing eucaryotic DNA sequences. J Virol 51: 595-603.

Lam P, Hui KM, Wang Y, Allen PD, Louis DN, Yuan CJ, Breakefield XO 2002. Dynamics of transgene expression in human glioblastoma cells mediated by herpes simplex virus/adeno-associated virus amplicon vectors. Hum Gene Ther 13: 2147-2159.

Larin Z, Mejia JE 2002. Advances in human artificial chromosome technology. Trends Genet 18: 313-319.

Lauterbach H, Ried C, Epstein AL, Marconi P, Brocker T 2005. Reduced immune responses after vaccination with a recombinant herpes simplex virus type 1 vector in the presence of antiviral immunity. J Gen Virol 86: 2401-2410.

Lim F, Hartley D, Starr P, Lang P, Song S, Yu L, Wang Y, Geller AI 1996. Generation of high-titer defective HSV-1 vectors using an IE 2 deletion mutant and quantitative study of expression in cultured cortical cells. Biotechniques 20: 460-469.

Liu Q, Perez CF, Wang Y 2006. Efficient site-specific integration of large transgenes by an enhanced herpes simplex virus/adenoassociated virus hybrid amplicon vector. J Virol 80: 1672-1679.

Logvinoff C, Epstein AL 2000. Genetic engineering of herpes simplex virus and vector genomes carrying loxP sites in cells expressing Cre recombinase. Virology 267: 102-110. 
Lu B, Federoff HJ 1995. Herpes simplex virus type 1 amplicon vectors with glucocorticoid-inducible gene expression. Hum Gene Ther 6: 419-428.

Lufino MM, Edser PA, Wade-Martins R 2008. Advances in highcapacity extrachromosomal vector technology: episomal maintenance, vector delivery and transgene expression. Mol Ther 16: $1525-1538$.

Lufino MM, Manservigi R, Wade-Martins R 2007. An S/MAR-based infectious episomal genomic DNA expression vector provides long-term regulated functional complementation of LDLR deficiency. NAR 35: e98.

Moralli D, Simpson KM, Wade-Martins R, Monaco ZL 2006. A novel human artificial chromosome gene expression system using herpes simplex virus type 1 vectors. EMBO Rep 7: 911-918.

Mossman KL, MacGregor PF, Rozmus JJ, Goryachev AB, Edwrds AM, Smiley JR 2001. Herpes simplex virus triggers and then disarms a host antiviral response. J Virol 75: 750-758.

Mossman KL, Smiley JR 2002. Herpes simplex virus ICP0 and ICP34.5 counteract distinct interferon-induced barriers to virus replication. J Virol 76: 1995-1998.

Muller L, Saydam O, Saeki Y, Heid I, Fraefel C 2005. Gene transfer into hepatocytes mediated by herpes simplex virus-Epstein-Barr virus hybrid amplicons. $J$ Virol Methods 123: 65-72.

Parrish E, Peltékian E, Dickson G, Epstein AL, Garcia L 1999. Cell engineering for muscle gene therapy: extemporaneous production of retroviral vector packaging macrophages using defective herpes simplex virus type 1 vectors harbouring gag, pol, env genes. Cytotechnology 30: 173-180.

Pechan PA, Fotaki M, Thompson RL, Dunn R, Chase M, Chiocca EA, Breakefield XO 1996. A novel 'piggyback' packaging system for herpes simplex virus amplicon vectors. Hum Gene Ther 7: 2003-2013.

Rasmussen M, Kong L, Zhang GR, Liu M, Wang X, Szabo G, Curthoys NP, Geller AI 2007. Glutamatergic or GABAergic neuron-specific, long-term expression in neocortical neurons from helper virus-free HSV-1 vectors containing the phosphate-activated glutaminase, vesicular glutamate transporter-1, or glutamic acid decarboxylase promoter. Brain Res 1144: 19-32.

Saeki Y, Breakefield XO, Chiocca EA 2003. Improved HSV-1 amplicon packaging system using ICP27-deleted, oversized HSV-1 BAC DNA. Methods Mol Med 76: 51-60.

Saeki Y, Fraefel C, Ichikawa T, Breakefield XO, Chiocca EA 2001. Improved helper virus-free packaging system for HSV amplicon vectors using an ICP27-deleted, oversized HSV-1 DNA in a bacterial artificial chromosome. Mol Ther 3: 591-601.

Saeki Y, Ichikawa T, Saeki A, Chiocca EA, Tobler K, Ackermann M, Breakefield XO, Fraefel C 1998. Herpes simplex virus type 1 DNA amplified as bacterial artificial chromosome in Escherichia coli: rescue of replication-competent virus progeny and packaging of amplicon vectors. Hum Gene Ther 9: 2787-2794.

Samady L, Costigliola E, MacCormac L, McGrath Y, Cleverley S, Lilley CE, Smith J, Latchman DS, Chain B, Coffin RS 2003. Deletion of the virion host shutoff protein (vhs) from herpes simplex virus (HSV) relieves the viral block to dendritic cell activation: potential of vhs- HSV vectors for dendritic cell-mediated immunotherapy. $J$ Virol 77: 3768-3776.

Santos K, Duke CMP, Dewhurst S 2006. Amplicons as vaccine vectors. Curr Gene Ther 6: 383-392.

Savard N, Cosset FL, Epstein AL 1997. Defective herpes simplex virus type 1 vectors harboring gag, pol and env genes can be used to rescue defective retrovirus vectors. J Virol 71: 4111-4117.
Sena-Esteves M, Saeki Y, Camp SM, Chiocca EA, Breakefield XO 1999. Single-step conversion of cells to retrovirus vector producers with herpes simplex virus-Epstein-Barr virus hybrid amplicons. J Virol 73: 10426-10439.

Shah K, Breakefield XO 2006. HSV amplicon vectors for cancer therapy. Curr Gene Ther 6: 361-370.

Song S, Wang Y, Bak SY, Lang P, Ullrey D, Neve RL, O'Malley KL, Geller AI 1997. An HSV-1 vector containing the rat tyrosine hydroxylase promoter enhances both long-term and cell type-specific expression in the midbrain. J Neurochem 68: 1792-1803.

Spaete RR, Frenkel N 1982. The herpes simplex virus amplicon: a new eucaryotic defective-virus cloning-amplifying vector. Cell 30: 295-304.

Spaete RR, Frenkel N 1985. The herpes simplex virus amplicon: analyses of cis-acting replication functions. Proc Natl Acad Sci USA 82: 694-698.

Stavropoulos TA, Strathdee CA 1998. An enhanced packaging system for helper-dependent herpes simplex virus vectors. $J$ Virol 72: 7137-7143.

Suzuki M, Chiocca EA, Saeki Y 2007. Early STAT1 activation after systemic delivery of HSV amplicon vectors suppresses transcription of the vector-encoded transgene. Mol Ther 15: 2017-2026.

Suzuki M, Chiocca EA, Saeki Y 2008. Stable transgene expression from HSV amplicon vectors in the brain: potential involvement of immunoregulatory signals. Mol Ther 16: 1727-1736.

Suzuki M, Kasai K, Saeki Y 2006. Plasmid DNA sequences present in conventional herpes simplex virus amplicon vectors cause rapid transgene silencing by forming inactive chromatin. J Virol 80 : 3293-3300.

Tsimbouri P, Drotar ME, Coy JL, Wilson JB 2002. bcl-xL and RAG genes are induced and the response to IL-2 enhanced in EmuEBNA-1 transgenic mouse lymphocytes. Oncogene 21: 5182-5187.

Tsitoura E, Georgopoulou U, Mavromara P 2006. HSV-1 based amplicon vectors as an alternative system for the expression of functional HCV proteins. Curr Gene Ther 6: 393-398.

Tsitoura E, Lucas M, Revol-Guyot V, Epstein AL, Manservigi R, Mavromara P 2002. Expression of hepatitis C virus envelope glycoproteins by herpes simplex virus type 1-based amplicon vectors. J Gen Virol 83: 561-566.

Tyler CM, Wuertzer CA, Bowers WJ, Federoff HJ 2006. HSV amplicons: neuro applications. Curr Gene Ther 6: 337-350.

Vlazny DA, Frenkel N 1981. Replication of herpes simplex virus DNA: localization of replication recognition signals within defective virus genomes. Proc Natl Acad Sci USA 78: 742-746.

Wade-Martins R, Saeki Y, Chiocca EA 2003. Infectious delivery of a 135-kb LDLR genomic locus leads to regulated complementation of low-density lipoprotein receptor deficiency in human cells. Mol Ther 7: 604-612.

Wade-Martins R, Smith ER, Tyminski E, Chiocca EA, Saeki Y 2001. An infectious transfer and expression system for genomic DNA loci in human and mouse cells. Nat Biotechnol 19: 1067-1070.

Wang S, Di S, Young WB, Jacobson C, Link CJ Jr 1997. A novel herpesvirus amplicon system for in vivo gene delivery. Gene Ther 4: 1132-1141.

Wang S, Petravicz J, Breakefield XO 2003. Single HSV-amplicon vector mediates drug-induced gene expression via dimerizer system. Mol Ther 7: 790-800.

Wang S, Vos JM 1996. A hybrid herpesvirus infectious vector based on Epstein-Barr virus and herpes simplex virus type 1 for gene transfer into human cells in vitro and in vivo. J Virol 70: 8422-843 
Wang Y, Camp SM, Niwano M, Shen X, Bakowska JC, Breakefield XO, Allen PD 2002. Herpes simplex virus type 1/adeno-associated virus rep $(+)$ hybrid amplicon vector improves the stability of transgene expression in human cells by site-specific integration. $J$ Virol 76: 7150-7162.

Whitley RJ 2001. Herpes simplex viruses. In DM Knipe, PM Howley (eds.), Fields Virology, Lippincott Williams \& Wilkins, Philadelphia, p. 2461-2510.

York IA, Roop C, Andrews DW, Riddell SR, Graham FL, Johnson DC
1994. A cytosolic herpes simplex virus protein inhibits antigen presentation to CD8+ T lymphocytes. Cell 77: 525-535.

Zaupa C, Revol-Guyot V, Epstein AL 2003. Improved packaging system for generation of high-level noncytotoxic HSV-1 amplicon vectors using Cre-loxP site-specific recombination to delete the packaging signals of defective helper genomes. Hum Gene Ther 14: 1049-1063.

Zhang X, O'Shea H, Entwisle C, Boursnell M, Efstathiou S, Inglis S 1998. An efficient selection system for packaging herpes simplex virus amplicons. J Gen Virol 79: 125-131. 